\title{
CO-BLUMBERG SPACES
}

\author{
J. B. BROWN AND Z. PIOTROWSKI
}

\begin{abstract}
A pair ( $X, Y)$ of topological spaces is said to be a Blumberg pair ("BP") if for every $f: X \rightarrow Y$, there exists a dense subset $D$ of $X$ such that $f \mid D$ is continuous. $X$ is a Blumberg space if $(X, R)$ is BP, where $R$ denotes the reals. $Y$ is co-Blumberg if $(R, Y)$ is BP. We survey the literature concerning the relationships between Blumberg spaces and Baire spaces and then study the relationships between co-Blumberg spaces and separability properties.
\end{abstract}

1. Blumberg spaces. Henry Blumberg proved that $(R, R)$ is $\mathrm{BP}$ in [B122], and there has been quite a bit of research along these lines since then. See [Bn83] for a survey with a fairly extensive bibliography.

We adopt the following notational convention: $\omega=\omega_{0}=\boldsymbol{\aleph}_{0}, \Omega=\omega_{1}=\boldsymbol{\aleph}_{1}$, and $c=2^{\omega}$. Let $n$ denote an infinite cardinal, and consider the following properties of topological spaces $X$, which are known to be closely related:

(1) no open set is the union of $n$ or fewer nowhere dense sets,

(2) the intersection of $n$ or fewer dense open sets is dense,

(3) $(X, C)$ is BP for every space $C$ with $|C|=n$,

(4) $(X, \operatorname{disc}(n))$ is $\mathrm{BP}$, where $\operatorname{disc}(n)$ is the discrete space with cardinality $n$,

(5) for every decomposition $P$ of $X$ (collection of disjoint subsets of $X$ with union $X$ ) of cardinality $\leqq n$, there exists a dense subset $D$ of $X$ such that for every $A \in P$, $A \cap D$ is open in $D$,

(6) $X$ is a Blumberg space,

(7) $\left(X, B^{n}\right)$ is BP ( $B$ denotes the binary space $\{0,1\}$ with discrete topology),

(8) for every cover $P$ of $X$ (with arbitrary sets) of cardinality $\leqq n$, there exists a dense subset $D$ of $X$ such that for every $A \in P, A \cap D$ is open in $D$.

(9) $(X, Y)$ is BP for every $Y$ with $w(Y) \leqq n$, where $w(Y)$ is the "weight" of $Y$ or the minimal cardinality of a basis for $Y$.

(10) for every topology $P$ on $X$ of weight $\leqq n$, there exists a subset $D$ of $X$, dense in $X$ in the original topology $T$, such that for every $A \in P, A \cap D$ is $T$-open in $D$.

For $n=\omega,(1)=(2)$ is the definition of a Baire space, and it was shown in [BdGo60] that for metric spaces $X$, Baire spaces and Blumberg spaces are the same. It was shown in [Bd62] that for metric spaces $X$ and $Y,(X, Y)$ is BP if and only if no

Received by the editors September 28, 1984 and, in revised form, April 2, 1985. Presented at the 7th Symposium on Real Analysis (sponsored by NSF) held at the University of California, Santa Barbara, March 20-23, 1984.

1980 Mathematics Subject Classification. Primary 54C30; Secondary 26A15.

Key words and phrases. Blumberg's theorem, Baire space, separability. 
open set in $X$ is the union of $w(Y)$ or fewer nowhere dense sets. It follows that $(2)=(9)$ for metric spaces $X$ and $Y$ and arbitrary $n$. We now know from the results of [StVo67] that this has limited applicability for metric spaces, because any metric space without isolated points is the union of $c$ or fewer nowhere dense sets (also see [Bg77] and [KuSz77]). It follows that if $X$ and $Y$ are metric and $w(Y)$ is greater than or equal to $c$, then $(X, Y)$ will be BP only if the set of isolated points of $X$ is dense in $X$. (Such spaces will be termed "trivial".) In this case, $(X, Z)$ will be BP for every space $Z$. For topological spaces $X$, the Blumberg property is strictly stronger than the Baire property. The examples in [Le73] and [We77] satisfy (1) for $n=\omega$ and therefore for every infinite $n$, but do not satisfy (7) for $n=\omega$, and therefore do not satisfy (7) for any infinite $n$. (1), (2), and (3) were shown to be equivalent for arbitrary $n$ in [Wh74], and (2), (4), and (5) were shown to be the same for arbitrary $n$ in [Sz77], where it was also shown that (6) and (7) are the same for $n=\omega$, that (7) and (8) are the same for arbitrary $n$, and that topological spaces with property (1) but without isolated points exist for arbitrary regular cardinals $n$ (in contrast to the metric case). In particular, it was shown that if $n$ is a regular cardinal, then $B^{n}$, with the "box" topology, satisfies (2). The question of whether nontrivial spaces with property (7) exist for large cardinals is still unsettled without imposition of extra set theoretic assumptions. It follows from [Sz77] and $\mathrm{GCH}$ that such will exist, but it is not known in ZFC if $\left(B^{n}, B^{n}\right)$ is BP for arbitrary $n$. It was shown in [Wh79] that (9) and (10) are equivalent for $n=\omega$, and the question was raised as to whether (6) implies (8) for $n=\omega$. This question was settled in the affirmative in [PiSz84], thus establishing the equivalence of (6) through (10) for $n=\omega$. The arguments for Theorem 1 and Corollary 2 of [PiSz84] carry over to arbitrary $n$. So to summarize, we have

THEOREM 1. For topological spaces $X$ and arbitrary cardinals $n,(1)$ through (5) are equivalent and (7) through (10) are equivalent and imply (1) through (5). For $n=\omega$, (6) and (7) are equivalent.

2. Co-Blumberg spaces. We now begin our study of the relationships between co-Blumberg spaces $Y$ and separability properties defined in terms of the weight, $w(Y)$, of $Y$ and related parameters such as the "spread", $s(Y)$, of $Y$, which is the supremum of the cardinalities of discrete subsets of $Y$.

THEOREM 2. $w(Y) \leqq \omega \rightarrow Y$ co-Blumberg $-\mathrm{CH} \rightarrow s(Y) \leqq \omega$.

Proof. The first implication follows from the equivalence of (6) and (9) for $n=\omega$. The second implication comes as follows. Under $\mathrm{CH}$, if $s(Y)>\omega$, it follows that there is a discrete subset $E$ of $Y$ with cardinality the same as that of $R$. Then, if $f$ is a 1-1 mapping of $R$ onto $E$, there could be no restriction of $f$ to a dense subset of $R$.

Consider the following parameters associated with a space $Y$. The "density character", $d(Y)$, of $Y$ is the minimum cardinality of a dense subset of $Y$. The "cellularity", $c(Y)$, of $Y$ is the supremum of the cardinalities of collections of 
disjoint open subsets of $Y$. It is well known (see [En68, Co71, or Ju71]) that

$$
\begin{aligned}
& d(Y) \leqq \omega \\
& \nearrow \quad \searrow
\end{aligned}
$$

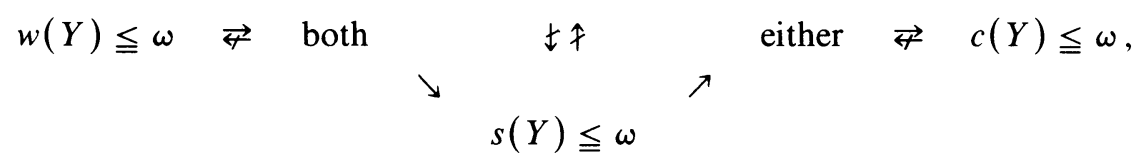

where "both" means that both $d(Y) \leqq \omega$ and $s(Y) \leqq \omega$ hold, and "either" means that at least one holds. The so-called Sorgenfrey line $S$ (Example 51 of [StSe78]), which consists of the reals topologized by the basis of half-open intervals $[a, b)$, satisfies "both" but not $w(Y) \leqq \omega$. The product $S^{2}$ (Example 84 of [StSe78]) satisfies $d(Y) \leqq \omega$ but not $s(Y) \leqq \omega$. The reals with the "countable complement extension topology" (Example 63 of [StSe78]), in which a set is open if and only if it is of the form $U \backslash C$ where $U$ is open in the Euclidean topology and $C$ is countable, satisfies $s(Y) \leqq \omega$ but not $d(Y) \leqq \omega$. Finally, the so-called "uncountable Fort space" (Example 24 of [StSe78]) satisfies $c(Y) \leqq \omega$ but not "either".

We now show that one cannot improve on Theorem 2 in trying to fit the co-Blumberg property into the above diagram (I) of implications.

EXAMPLE 1. "both" $\nrightarrow$ co-Blumberg.

Proof. The Sorgenfrey line, $S$, satisfies "both" but is not co-Blumberg. To see this, consider the identity function $f: R \rightarrow S$, and suppose $D$ is a dense subset of $R$ such that $f \mid D$ is continuous. Then, if $x \in D$, every neighborhood of $x$ contains an element $y$ of $D$ to the left of $x$, and $f(y)$ will not be in $[f(x), f(x)+1) \cdot f \mid D$ cannot be continuous.

EXAMPLE 2. co-Blumberg $\nrightarrow w(Y) \leqq \omega$.

Proof. One could use $R$ with the "co-finite" topology here (Example 19 of [StSe78]), but a better example is the "Arens-Fort" space (Example 26 of [StSe78]). This is a countable Hausdorff space which is not first countable, and therefore fails to satisfy $w(Y) \leqq \omega$. Since $Y$ is a countable space, it follows from the equivalence of (3) and (6) for metric $X$ that $Y$ is co-Blumberg.

Example 2 is described in ZFC. We will see in Example 4 that it follows from $\mathrm{CH}$ that co-Blumberg $\nrightarrow$ "both". First we point out that the $\mathrm{CH}$ assumption cannot be dropped from the second implication of Theorem 2 .

EXAMPle 3. (MA + not $\mathrm{CH})$ co-Blumberg $\nrightarrow c(Y) \leqq \omega$.

Proof. Assume (MA + not $\mathrm{CH}$ ) and let $Y=\operatorname{disc}(\Omega)$. $Y$ obviously fails to satisfy $c(Y) \leqq \omega$. Since MA implies (1) for $X=R$ and $n=\Omega$, it follows from the equivalence of (1) and (4) that $Y$ is co-Blumberg.

EXAMPLE 4. $(\mathrm{CH})$ co-Blumberg $\nrightarrow d(Y) \leqq \omega$.

Proof. This example must be nonseparable but must have countable spread. Most of the 38 nonseparable examples of [StSe78] have uncountable spread. We state with some certainty that none of the other nonseparable examples of [StSe78] will suffice here. In particular, the reals, $E$, with the "countable complement extension topology" (Example 63 of [StSe78]) is not co-Blumberg. To see this, let $f: R \rightarrow E$ be the identity function. If there is a dense subset $D$ of $R$ such that $f \mid D$ is continuous, 
there is a countable such $D$. But then, if $x \in D, f(x)$ is an isolated point in $f(D)$ with the relative topology of $E$. It follows that $f \mid D$ cannot be continuous.

A modification of this example which can be found as Example 6.3 of [Ju71] will serve here. Let $Z$ denote the reals and $\prec$ denote a well-ordering of $Z$ which has countable initial segments. As a basis for the topology $T$ of $Z$, take sets of the form $U_{x}$, where $U$ is open in the Euclidean topology, $x \in U$, and $U_{x}$ consists of the $U$ minus the elements of $U$ which precede $x$ in the ordering. It is easy to see that $Z$ fails to satisfy $d(Y) \leqq \omega$. The proof that $Z$ is co-Blumberg is rather involved, so we state this as a separate theorem.

\section{THEOREM 3. (CH) $Z$ is co-Blumberg.}

Proof. Let $f: R \rightarrow Z$, and let $U$ denote the Euclidean topology for $R$. We say that $M \subseteq R$ is "categorically dense" in an open set $Q \in U$ if $M \cap Q$ ' is second category for every open $Q^{\prime} \subseteq Q$. Assume there is an open set $Q \in U$ and an $x \in Q$ such that $f^{-1}(f(x)) \cap Q$ is categorically dense in $Q$. Well order such sets $Q$ as $\left\{Q_{\alpha}\right\}_{\alpha<\Omega}$, and for each $\alpha$, let $x_{\alpha}$ be such an $x$ that goes with $Q_{\alpha}$. Now, for each $\alpha$ such that $W_{\alpha}=Q_{\alpha} \backslash \mathrm{Cl}\left(U_{\beta<\alpha} Q_{\beta}\right)$ is nonempty, let $E_{\alpha}=f^{-1}\left(f\left(x_{\alpha}\right)\right) \cap W_{\alpha}$. Note that $D_{1}=U_{\alpha<\Omega} E_{\alpha}$ is dense in the open set $W=U_{\alpha<\Omega} W_{\alpha}$, and $f \mid D_{1}$ is continuous regardless of which topology is imposed on the range space $Z$. It should be noticed that it is the case that if $M$ is a second category subset of $R \backslash \mathrm{Cl}(W)$, then $f(M)$ is uncountable.

Now, let $Q=R \backslash \mathrm{Cl}(W)$. We will make use of the following well-known tool which is used in proving the standard Blumberg's Theorem.

Lemma. (L1): If $A$ is categorically dense in the open set $Q$, there exist an $x \in A$ and a subset $C$ of $A$, also categorically dense in $Q$ and containing $x$, such that $f \mid C$ is continuous at $x$ (where the topology on the range space is $U$ ); in fact, the set of such $x$ is also categorically dense in $Q$.

See $[\mathbf{B n} 71]$ for a proof of this assertion. Let $\left\{B_{1}, B_{2}, \ldots\right\}$ be a countable basis for the $U$-topology relativized to $Q$. Now, proceed as in the usual proof of Blumberg's Theorem. Let $A_{1}=Q$, and let $x_{1}$ and $A_{2}$ be the $x$ and $C$ of (L1), with $A=A_{1}$, where $x_{1}$ is chosen to be in $B_{1}$. Let $x_{2}$ and $A_{3}$, be the $x$ and $C$ of (L1), with $A=A_{2}$, where $x_{2}$ is chosen to be in $B_{2}$ and $x_{2}$ is chosen so that $f\left(x_{2}\right)$ is a successor of $f\left(x_{1}\right)$ in the ordering $\prec$. The steps taken in the first paragraph of this proof make this possible. Continue this process, making sure that $x_{n}$ is a chosen so that $f\left(x_{n}\right)$ is a successor of $f\left(x_{1}\right), f\left(x_{2}\right), \ldots$, and $f\left(x_{n-1}\right)$ for each $n$. The set $D_{2}=\left\{x_{1}, x_{2}, \ldots\right\}$ is dense in $Q$, and $f \mid D_{2}$ is continuous even in the restriction of the topology $T$ to the range $R$ of $f \mid D_{2}$. This is because for each $n$, an open set in this restricted topology containing $f\left(x_{n}\right)$ is the restriction of an open set in the Euclidean topology to this range minus a finite set. Since $f \mid D_{2}$ is clearly continuous when the topology is the Euclidean one on both domain and range, the result follows. $D_{1} \cup D_{2}$ is the required set, and the theorem is proved.

We now present some open problems related to the theorems and examples of this section. 
Problem 1. Avoid the $(\mathrm{CH})$ assumption in Example 4 or show that it is not possible to avoid this assumption.

Problem 2. It is clear from Theorem 2 and the equivalence of the conditions of (I) in the metric case that a metric space is co-Blumberg if and (under $\mathrm{CH}$ ) only if it is separable. Thus, Examples 1, 2, and 4 are nonmetrizable (indeed, Example 2 is not first countable and Example 4 is not regular). Might there be topological conditions to be imposed on $Y$ which are not strong enough to make the conditions of (I) equivalent but which would be strong enough to make one of the implications co-Blumberg $\rightarrow w(y) \leqq \omega$, co-Blumberg $\rightarrow d(Y) \leqq \omega$, or " both" $\rightarrow$ co-Blumberg hold?

Problem 3. Is $\left(B^{n}, B^{n}\right)$ BP for every infinite cardinal $n$ ?

Problem 4. Find analogous result to those presented here but for cardinals $n>\omega$, where "co-Blumberg" is replaced by " $\left(B^{n}, Y\right)$ is BP". Of course, this would not be a logical extension of the results of this section without the following.

THEOREM 4. $Y$ is co-Blumberg if and only if $\left(B^{\omega}, Y\right)$ is BP.

Proof. Unlike showing the equivalence of (6) and (7) for $n=\omega$, both implictions will require some argument. First assume $Y$ is co-Blumberg and let $f: B^{\omega} \rightarrow Y$. The set $M$ of points of $B^{\omega}$ which are not "endpoints" can be thought of as being imbedded by a homeomorphism $h$ into $R$ as the set $N$ of irrationals. Let $g: R \rightarrow Y$ be defined as follows: $g=f\left(h^{-1}\right)$ on $N$. Let $\left\{r_{1}, r_{2}, \ldots\right\}$ be a listing of the rationals. We can assume the space $Y$ is infinite and that there exists a nonrepeating sequence $y_{1}, y_{2}, \ldots$ of elements of $Y$ which either has no convergent subsequence or else converges to some element of $Y$ not in the sequence. Define $g$ on the rationals by $f\left(r_{n}\right)=y_{n}$. Since $Y$ is co-Blumberg, there exists a dense subset $D$ of $R$ such that $g \mid D$ is continuous. Because of the way $g$ is defined on the rationals, it follows that $D \cap\left\{r_{1}, r_{2}, \ldots\right\}$ is nowhere dense, so $D \cap N$ is dense in $R \cdot h^{-1}(D \cap N)$ is the required dense subset of $B^{\omega}$.

\section{REFERENCES}

[Bg77] K. E. Broughan, The intersection of a continuum of open dense sets, Bull. Austral. Math. Soc. 16 (1977), 267-272.

[BdGo60] J. C. Bradford and C. Goffman, Metric spaces in which Blumberg's theorem holds, Proc. Amer. Math. Soc.11 (1960), 667-670.

[Bd62] J. C. Bradford, Characterization of metric Blumberg pairs, unpublished manuscript.

[B122] H. Blumberg, New properties of all real functions, Trans. Amer. Math. Soc. 3 (1922), 113-128.

[Bn71] J. B. Brown, Metric spaces in which a strengthened form of Blumberg's theorem holds, Fund. Math. 71 (1971), 243-253.

[Bn83] Uariations on Blumberg's theorem, Real Analysis Exchange 9 (1983/84), 123-137.

[Co71] W. W. Comfort, A survey of cardinal invariants, General Topology Appl. 1 (1971), 163-199.

[En68] R. Engelking, Outline of general topology, North-Holland, Amsterdam, 1968.

[Ju71] I. Juhaz, Cardinal functions in topology, Math. Centre Tracts 34, Amsterdam, 1971.

[KuSz77] W.Kulpa and A. Szymanski, Decompositions into nowhere dense sets, Bull. Acad. Polon. Sci. 25 (1977), 37-39.

[Le73] R. Levy, A totally ordered Baire space for which Blumberg's theorem fails, Proc. Amer. Math. Soc. 41 (1973), 304.

[PiSz84] Z. Piotrowski and A. Szymanski, Concerning Blumberg's theorem, Houston J. Math. 10 (1984), $109-115$. 
[StSe78] L. A. Steen and J. A. Seebach, Jr., Counterexamples in topology, Springer-Verlag, 1978.

[StVo67] P. Stepanek and P. Vopenka, Decompositions of metric spaces into nowhere dense sets, Comment. Math. Univ. Carolin. 8 (1967), 389-394.

[Sz77] A. Szymanski, On m-Baire and m-Blumberg spaces, Proc. Conf. Topology and Measure II (Rostock-Warnemunde, GDR, 1977), Greifswald, 1980, Part 1, pp. 151-161.

[We77] W. A. R. Weiss, The Blumberg problem, Trans. Amer. Math. Soc. 230 (1977), 71-85.

[Wh74] H. E. White, Topological spaces in which Blumberg's theorem holds, Proc. Amer. Math. Soc. 44 (1974), 454-462.

[Wh79] Topological spaces in which Blumberg's theorem holds II, Illinois J. Math. 23 (1979), 464-468.

Department of Mathematics, Auburn University, Auburn, Alabama 36849

Department of Mathematics, Youngstown State University, Youngstown, Ohio 44555 